\title{
ON THE THREE-DIMENSIONAL PAULI EQUATION IN NONCOMMUTATIVE PHASE-SPACE
}

\author{
ILYAS HAOUAM
}

\author{
Université Frères Mentouri, Laboratoire de Physique Mathématique et de Physique Subatomique (LPMPS), \\ Constantine 25000, Algeria \\ correspondence: ilyashaouam@live.fr
}

\begin{abstract}
In this paper, we obtained the three-dimensional Pauli equation for a spin- $1 / 2$ particle in the presence of an electromagnetic field in a noncommutative phase-space as well as the corresponding deformed continuity equation, where the cases of a constant and non-constant magnetic fields are considered. Due to the absence of the current magnetization term in the deformed continuity equation as expected, we had to extract it from the noncommutative Pauli equation itself without modifying the continuity equation. It is shown that the non-constant magnetic field lifts the order of the noncommutativity parameter in both the Pauli equation and the corresponding continuity equation. However, we successfully examined the effect of the noncommutativity on the current density and the magnetization current. By using a classical treatment, we derived the semi-classical noncommutative partition function of the three-dimensional Pauli system of the one-particle and N-particle systems. Then, we employed it for calculating the corresponding Helmholtz free energy followed by the magnetization and the magnetic susceptibility of electrons in both commutative and noncommutative phase-spaces. Knowing that with both the three-dimensional Bopp-Shift transformation and the Moyal-Weyl product, we introduced the phase-space noncommutativity in the problems in question.
\end{abstract}

Keywords: 3-D noncommutative phase-space, Pauli equation, deformed continuity equation, current magnetization, semi-classical partition function, magnetic susceptibilit.

\section{INTRODUCTION}

It is well known that the Dirac equation is the relativistic wave equation that describes the motion of the spin-1/2 fermions and the Pauli equation, which is a topic of great interest in physics, is the non-relativistic wave equation describing it 1-4]. It is relative to the explanation of many experimental results, and its probability current density changed to include an additional spin-dependent term recognized as the spin current [5] 7]. Pauli equation is shown in 812 as the non-relativistic limit of the Dirac equation. Historically, Pauli (1927) presented his famous spin matrices [13] for adjusting the non-relativistic Schrödinger equation to account for Goudsmit-Uhlenbeck's hypothesis (1925) [14, 15. Therefore, he applied an ansatz for adding a phenomenological term to the ordinary non-relativistic Hamiltonian in the presence of an electromagnetic field, the interaction energy of a magnetic field and electronic magnetic moment relative to the intrinsic spin angular momentum of the electron. Describing this spin angular momentum through the spin matrices requires replacing the complex scalar wave function by a two-component spinor wave function in the wave equation. Since then, the study of the Pauli equation became a matter of considerable attention.

In 1928, when Dirac presented his relativistic free wave equation in addition to the minimal coupling replacement to include electromagnetic interactions [16, he showed that his equation contained a term involving the electron magnetic moment interacting with a magnetic field, which was the same one inserted by hand in Pauli's equation. After that, it became common to count an electron spin as a relativistic phenomenon, and the corresponding spin- $1 / 2$ term could be inserted into the spin-0 non-relativistic Schrödinger equation as will be discussed in this article to see how this is possible. However, motivated by attempts to understand the string theory and describe quantum gravitation using noncommutative geometry and by trying to draw a considerable attention to the phenomenological implications, we focus on studying the problem of a non-relativistic spin- $1 / 2$ particle in the presence of an electromagnetic field within 3-dimensional noncommutative phase-space.

As a mathematical theory, noncommutative geometry is by now well established, although at first, its progress has been narrowly restricted to some branches of physics such as quantum mechanics. However, recently, the noncommutative geometry has become a topic of great interest [17- 23]. It has been finding applications in many sectors of physics and has rapidly become involved in them, continuing to promote fruitful ideas and the search for a better understanding. Such as in the quantum gravity [24; the standard model of fundamental interactions [25]; as well as in the string theory [26]; and its implication in Hopf algebras [27] gives the Connes-Kreimer 
Hopf algebras [28 30] etc. There are many papers devoted to the study of such various aspects especially in quantum field theory [31 33] and quantum mechanics [34-36].

This paper is organized as follows. In section 2, we present an analysis review of noncommutative geometry, in particular both the three-dimensional Bopp-Shift transformation and the Moyal-Weyl product. In section 3, we investigate the three-dimensional Pauli equation in the presence of an electromagnetic field and the corresponding continuity equation. Furthermore, we derived the current magnetization term in the deformed continuity equation. Section 4 is devoted to calculating the semi-classical noncommutative partition function of the Pauli system of the one-particle and N-particle systems. Consequently, we obtain the corresponding magnetization and the magnetic susceptibility through the Helmholtz free energy, all in both commutative and noncommutative phase-spaces and within a classical limit. Therefore, concluding with some remarks.

\section{REVIEW OF NONCOMMUTATIVE ALGEBRA}

Firstly, we present the most essential formulas of noncommutative algebra [36. It is well known that at very small scales such as the string scale, the position coordinates do not commute with each other, neither do the momenta.

Let us accept, in a d-dimensional noncommutative phase-space, the operators of coordinates and momenta $x_{i}^{n c}$ and $p_{i}^{n c}$, respectively. The noncommutative formulation of quantum mechanics corresponds to the following Heisenberg-like commutation relations

$$
\left[x_{\mu}^{n c}, x_{\nu}^{n c}\right]=i \Theta_{\mu \nu}, \quad\left[p_{\mu}^{n c}, p_{\nu}^{n c}\right]=i \eta_{\mu \nu}, \quad\left[x_{\mu}^{n c}, p_{\nu}^{n c}\right]=i \tilde{\hbar} \delta_{\mu \nu} \quad,(\mu, \nu=1, . . d)
$$

the effective Planck constant is the deformed Planck constant, which is given by

$$
\tilde{\hbar}=\alpha \beta \hbar+\frac{\operatorname{Tr}[\Theta \eta]}{4 \alpha \beta \hbar}
$$

where $\frac{\operatorname{Tr}[\Theta \eta]}{4 \alpha \beta \hbar} \ll 1$ is the condition of consistency in quantum mechanics. $\Theta_{\mu \nu}, \eta_{\mu \nu}$ are constant antisymmetric $d \times d$ matrices and $\delta_{\mu \nu}$ is the identity matrix.

It is shown that $x_{i}^{n c}$ and $p_{i}^{n c}$ can be represented in terms of coordinates $x_{i}$ and momenta $p_{j}$ in usual quantum mechanics through the so-called generalized Bopp-shift as follows 34

$$
x_{\mu}^{n c}=\alpha x_{\mu}-\frac{1}{2 \alpha \hbar} \Theta_{\mu \nu} p_{\nu}, \text { and } \quad p_{\mu}^{n c}=\beta p_{\mu}+\frac{1}{2 \beta \hbar} \eta_{\mu \nu} x_{\nu}
$$

with $\alpha=1-\frac{\Theta \eta}{8 \hbar^{2}}$ and $\beta=\frac{1}{\alpha}$ being scaling constants.

To the 1rst order of $\Theta$ and $\eta$, in the calculations we take $\alpha=\beta=1$, so the Equations (3) 2 become

$$
x_{\mu}^{n c}=x_{\mu}-\frac{1}{2 \hbar} \Theta_{\mu \nu} p_{\nu}, \quad p_{\mu}^{n c}=p_{\mu}+\frac{1}{2 \hbar} \eta_{\mu \nu} x_{\nu}, \text { and } \tilde{\hbar}=\hbar+\frac{\operatorname{Tr}[\Theta \eta]}{4 \hbar} .
$$

If the system in which we study the effects of noncommutativity is three-dimensional, we limit ourselves to the following noncommutative algebra

$$
\left[x_{j}^{n c}, x_{k}^{n c}\right]=i \frac{1}{2} \epsilon_{j k l} \Theta_{l}, \quad\left[p_{j}^{n c}, p_{k}^{n c}\right]=i \frac{1}{2} \epsilon_{j k l} \eta_{l}, \quad\left[x_{j}^{n c}, p_{k}^{n c}\right]=i\left(\hbar+\frac{\Theta \eta}{4 \hbar}\right) \delta_{j k} \quad,(j, k, l=1,2,3)
$$

$\Theta_{l}=(0,0, \Theta), \eta_{l}=(0,0, \eta)$ are the real-valued noncommutative parameters with the dimension of length ${ }^{2}$, momentum $^{2}$ respectively, they are assumed to be extremely small. And $\epsilon_{j k l}$ is the Levi-Civita permutation tensor. Therefore, we have

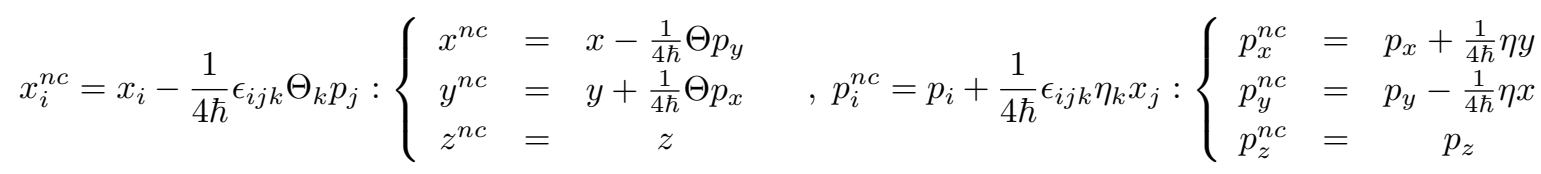

In noncommutative quantum mechanics, it is quite possible that we replace the usual product with the Moyal-Weyl $(\star)$ product, then the quantum mechanical system will simply become the noncommutative quantum mechanical system. Let $\mathcal{H}(x, p)$ be the Hamiltonian operator of the usual quantum system, then the corresponding Schrödinger equation on noncommutative quantum mechanics is typically written as

$$
\mathcal{H}(x, p) \star \psi(x, p)=E \psi(x, p) .
$$


The definition of Moyal-Weyl product between two arbitrary functions $f(x, p)$ and $g(x, p)$ in phase-space is given by 37

$$
\begin{gathered}
(f \star g)(x, p)=\exp \left[\frac{i}{2} \Theta_{a b} \partial_{x_{a}} \partial_{x_{b}}+\frac{i}{2} \eta_{a b} \partial_{p_{a}} \partial_{p_{b}}\right] f\left(x_{a}, p_{a}\right) g\left(x_{b}, p_{b}\right)=f(x, p) g(x, p) \\
+\sum_{n=1} \frac{1}{n !}\left(\frac{i}{2}\right)^{n} \Theta^{a_{1} b_{1}} \ldots \Theta^{a_{n} b_{n}} \partial_{a_{1}}^{x} \ldots \partial_{a_{k}}^{x} f(x, p) \partial_{b_{1}}^{x} \ldots \partial_{b_{k}}^{x} g(x, p) \\
+\sum_{n=1} \frac{1}{n !}\left(\frac{i}{2}\right)^{n} \eta^{a_{1} b_{1}} \ldots \eta^{a_{n} b_{n}} \partial_{a_{1}}^{p} \ldots \partial_{a_{k}}^{p} f(x, p) \partial_{b_{1}}^{p} \ldots \partial_{b_{k}}^{p} g(x, p)
\end{gathered}
$$

with $f(x, p)$ and $g(x, p)$ assumed to be infinitely differentiable. If we consider the case of a noncommutative space, the definition of Moyal-Weyl product will be reduced to [38]

$$
(f \star g)(x)=\exp \left[\frac{i}{2} \Theta_{a b} \partial_{x_{a}} \partial_{x_{b}}\right] f\left(x_{a}\right) g\left(x_{b}\right)=f(x) g(x)+\sum_{n=1} \frac{1}{n !}\left(\frac{i}{2}\right)^{n} \Theta^{a_{1} b_{1}} \ldots \Theta^{a_{n} b_{n}} \partial_{a_{1}} \ldots \partial_{a_{k}} f(x) \partial_{b_{1}} \ldots \partial_{b_{k}} g(x)
$$

Due to the nature of the $\star$ product, the noncommutative field theories for low-energy fields $\left(E^{2} \lesssim 1 / \Theta\right)$ at a classical level are completely reduced to their commutative versions. However, this is just the classical result and quantum corrections always reveal the effects of $\Theta$ even at low-energies.

On a noncommutative phase-space the $\star$ product can be replaced by a Bopp's shift, i.e., the $\star$ product can be changed into the ordinary product by replacing $\mathcal{H}(x, p)$ with $\mathcal{H}\left(x^{n c}, p^{n c}\right)$. Thus, the corresponding noncommutative Schrödinger equation can be written as

$$
\mathcal{H}(x, p) \star \psi(x, p)=\mathcal{H}\left(x_{i}-\frac{1}{2 \hbar} \Theta_{i j} p_{j}, p_{\mu}+\frac{1}{2 \hbar} \eta_{\mu \nu} x_{\nu}\right) \psi=E \psi .
$$

Note that $\Theta$ and $\eta$ terms can always be treated as a perturbation in quantum mechanics.

If $\Theta=\eta=0$, the noncommutative algebra reduces to the ordinary commutative one.

\section{Pauli equation in noncommutative Phase-SPaCe}

\subsection{Formulation of noncommutative Pauli equation}

The Pauli equation is the formulation of the Schrödinger equation for spin- $1 / 2$ particles, which was formulated by W. Pauli in 1927. It takes into account the interaction of the particle's spin with an electromagnetic field. In other words, it is the nonrelativistic limit of the Dirac equation. Furthermore, the Pauli equation could be extracted from other relativistic higher spin equations such as the DKP equation considering the particle interacting with an electromagnetic field [37. The nonrelativistic Schrödinger equation that describes an electron in interaction with an electromagnetic potential $\left(A_{0}, \vec{A}\right)\left(\hat{\vec{p}}\right.$ is replaced with $\hat{\vec{\pi}}=\hat{\vec{p}}-\frac{e}{c} \vec{A}$ and $\hat{E}$ with $\left.\hat{\epsilon}=i \hbar \frac{\partial}{\partial t}-e \phi\right)$ is

$$
\frac{1}{2 m}\left(\hat{\vec{p}}-\frac{e}{c} \vec{A}(r)\right)^{2} \psi(r, t)+e \phi(r) \psi(r, t)=i \hbar \frac{\partial}{\partial t} \psi(r, t)
$$

where $\hat{\vec{p}}=i \hbar \vec{\nabla}$ is the momentum operator, $m, e$ are the mass and charge of the electron, and $c$ is the speed of light. $\psi(r, t)$ is the Schrödinger's scalar wave function. The appearance of real-valued electromagnetic Coulomb and vector potentials, $\phi(\vec{r}, t)$ and $\vec{A}(\vec{r}, t)$, is a consequence of using the gauge-invariant minimal coupling assumption to describe the interaction with the external magnetic and electric fields defined by

$$
\vec{E}=-\vec{\nabla} \phi-\frac{1}{c} \frac{\partial \vec{A}}{\partial t}, \quad \vec{B}=\vec{\nabla} \times \vec{A}
$$

However, the electron gains potential energy when the spin interacts with the magnetic field, therefore, the Pauli equation of an electron with a spin is given by [1, 8]

$$
\frac{1}{2 m}(\vec{\sigma} \cdot \hat{\vec{\pi}})^{2} \psi(r, t)+e \phi \psi(r, t)=\frac{1}{2 m}\left(\hat{\vec{p}}-\frac{e}{c} \vec{A}\right)^{2} \psi(r, t)+e \phi \psi(r, t)+\mu_{B} \vec{\sigma} \cdot \vec{B} \psi(r, t)=i \hbar \frac{\partial}{\partial t} \psi(r, t)
$$

where $\psi(r, t)=\left(\begin{array}{ll}\psi_{1} & \psi_{2}\end{array}\right)^{T}$ is the spinor wave function, which replaces the scalar wave function. With $\mu_{B}=\frac{|e| \hbar}{2 m c}=9.27 \times 10^{-24} J T^{-1}$ being the Bohr's magneton, $\vec{B}$ is the applied magnetic field vector and $\mu_{B} \vec{\sigma}$ represents the magnetic moment. $\vec{\sigma}$ 's being the three Pauli matrices $(\operatorname{Tr} \vec{\sigma}=0)$, which obey the following algebra

$$
\left[\sigma_{i}, \sigma_{j}\right]=2 i \epsilon_{i j k} \sigma_{k}
$$




$$
\begin{gathered}
\sigma_{i} \sigma_{j}=\delta_{i j} I+i \sum_{k} \epsilon_{i j k} \sigma_{k}, \\
(\vec{\sigma} \cdot \hat{\vec{a}})(\vec{\sigma} \cdot \hat{\vec{b}})=\hat{\vec{a}} \cdot \hat{\vec{b}}+i \vec{\sigma} \cdot(\hat{\vec{a}} \times \hat{\vec{b}}),
\end{gathered}
$$

$\hat{\vec{a}}, \hat{\vec{b}}$ are any two vector operators that commute with $\vec{\sigma}$. It must be emphasized that the third term of equation (13) is the Zeeman term, which is generated automatically by using feature (16) with a correct g-factor of $g=2$ as reduced in the Bohr's magneton rather than being introduced by hand as a phenomenological term, as is usually done.

The Pauli equation in a noncommutative phase-space is

$$
\mathcal{H}\left(x^{n c}, p^{n c}\right) \psi\left(x^{n c}, t\right)=\mathcal{H}\left(x, p^{n c}\right) \star \psi(x, t)=e^{\frac{i}{2} \Theta_{a b} \partial_{x_{a}} \partial_{x_{b}}} \mathcal{H}\left(x_{a}, p^{n c}\right) \psi\left(x_{b}, t\right)=i \hbar \frac{\partial}{\partial t} \psi(x, t) .
$$

Here we achieved the noncommutativity in space using Moyal $\star$ product, and then the noncommutativity in phase through Bopp-shift. Using equation (9), we have

$$
\begin{aligned}
& \mathcal{H}\left(x^{n c}, p^{n c}\right) \psi\left(x^{n c}, t\right)= \\
& \quad=\left\{\mathcal{H}\left(x, p^{n c}\right)+\frac{i}{2} \Theta^{a b} \partial_{a} \mathcal{H}\left(x, p^{n c}\right) \partial_{b}+\sum_{n=2} \frac{1}{n !}\left(\frac{i}{2}\right)^{n} \Theta^{a_{1} b_{1}} \ldots \Theta^{a_{n} b_{n}} \partial_{a_{1}} \ldots \partial_{a_{k}} \mathcal{H}\left(x, p^{n c}\right) \partial_{b_{1}} \ldots \partial_{b_{k}}\right\} \psi
\end{aligned}
$$

In the case of a constant real magnetic field $\vec{B}=(0,0, B)=B \vec{e}_{3}$ oriented along the axis (Oz), which is often referred to as the Landau system. We have the following symmetric gauge

$$
\vec{A}=\frac{\vec{B} \times \vec{r}}{2}=\frac{B}{2}(-y, x, 0), \text { with } A_{0}(x)=e \phi=0 .
$$

Therefore, the derivations in the equation (18) shut down approximately in the first-order of $\Theta$, then the noncommutative Pauli equation in the presence of a uniform magnetic field can be written as follows

$$
\mathcal{H}\left(x, p^{n c}\right) \star \psi(x)=\left\{\frac{1}{2 m}\left(\vec{p}^{n c}-\frac{e}{c} \vec{A}(x)\right)^{2}+\mu_{B} \vec{\sigma} \cdot \vec{B}+\frac{i e}{4 m c} \Theta^{a b} \partial_{a}\left(\frac{e}{c} \vec{A}^{2}-2 \vec{p}^{n c} \cdot \vec{A}\right) \partial_{b}\right\} \psi(x)+0\left(\Theta^{2}\right),
$$

with $\left[\vec{p}^{n c}, \vec{A}\right]=0$. We now make use of the Bopp-shift transformation $[4$, in the momentum operator to obtain

$$
\begin{gathered}
\mathcal{H}\left(x^{n c}, p^{n c}\right) \psi\left(x^{n c}, t\right)=\left\{\frac{1}{2 m}\left(p_{i}+\frac{1}{2 \hbar} \eta_{i j} x_{j}-\frac{e}{c} A_{i}\right)^{2}+\mu_{B} \vec{\sigma} \cdot \vec{B}\right. \\
\left.-\frac{i e}{4 m c} \Theta^{a b} \partial_{a}\left(2\left(p_{i}+\frac{1}{2 \hbar} \eta_{i j} x_{j}\right) A_{i}-\frac{e}{c} \vec{A}^{2}\right) \partial_{b}\right\} \psi(x, t)=i \hbar \frac{\partial}{\partial t} \psi(x, t),
\end{gathered}
$$

we rewrite the above equation in a more compact form

$$
\begin{gathered}
\mathcal{H}\left(x, p^{n c}\right) \star \psi(x, t)=\left\{\frac{1}{2 m}\left(\vec{p}-\frac{e}{c} \vec{A}\right)^{2}-\frac{1}{2 m}(\vec{x} \times \vec{p}) \cdot \vec{\eta}-\frac{1}{2 m} \frac{e}{c \hbar}(\vec{x} \times \vec{A}(x)) \cdot \vec{\eta}\right. \\
\left.+\frac{1}{8 m \hbar^{2}} \eta_{i j} \eta_{\alpha \beta} x_{j} x_{\beta}+\mu_{B} \vec{\sigma} \cdot \vec{B}+\frac{e}{4 \hbar m c}\left(\vec{\nabla}\left(2 \vec{p} \cdot \vec{A}-\frac{1}{2 \hbar}(\vec{x} \times \vec{A}(x)) \cdot \vec{\eta}-\frac{e}{c} \vec{A}^{2}\right) \times \vec{p}\right) \cdot \vec{\Theta}\right\} \psi(x, t) .
\end{gathered}
$$

We restrict ourselves only to the first-order of the parameter $\eta$. The only reason behind this consideration is the balance with the noncommutativity in the space considered in the case of a constant magnetic field. Thus we now have

$$
\begin{aligned}
& \mathcal{H}\left(x, p^{n c}\right) \star \psi(x, t)=\left\{\frac{1}{2 m}\left(\vec{p}-\frac{e}{c} \vec{A}\right)^{2}-\frac{1}{2 m} \vec{L} \cdot \vec{\eta}-\frac{e}{2 m c \hbar}(\vec{x} \times \vec{A}(x)) \cdot \vec{\eta}+\mu_{B} \vec{\sigma} \cdot \vec{B}\right. \\
& \left.\left.+\frac{e}{4 m c \hbar}\left(\vec{\nabla}\left(2 \vec{p} \cdot \vec{A}(x)-\frac{1}{2 \hbar}(\vec{x} \times \vec{A}(x)) \cdot \vec{\eta}-\frac{e}{c} \vec{A}^{2}\right) \times \vec{p}\right) \cdot \vec{\Theta}\right\} \psi(x, t)\right)=i \hbar \frac{\partial}{\partial t} \psi(x, t) .
\end{aligned}
$$

The existence of a Pauli equation for all orders of the $\Theta$ parameter is explicitly relative to the magnetic field.

In the case of a non-constant magnetic field, we introduce a function depending on $x$ in the Landau gauge as $A_{2}=x B f(x)$, which gives us a non-constant magnetic field. The magnetic field can be easily calculated using the second equation of equation $[12$ as follows [33]

$$
\vec{B}(x)=B f(x) \vec{e}_{3} .
$$

If we specify $f(x)$, we obtain different classes of the non-constant magnetic field. If we take $f(x)=1$ in this case, we get a constant magnetic field.

Having the equation (23) on hand, we calculate the probability density and the current density. 


\subsection{Deformed CONTINUITy EQUATION}

In the following we calculate the current density, which results from the Pauli equation (23) that describes a system of two coupled differential equations for $\psi_{1}$ and $\psi_{2}$.

By putting

$$
\mathcal{Q}_{\eta}=\mathcal{Q}_{\eta}^{*}=(\vec{x} \times \vec{A}(x)) \cdot \vec{\eta}, \quad \mathcal{Q}_{\Theta}=\left(\vec{\nabla}\left(2 \vec{p} \cdot \vec{A}(x)-\frac{1}{2 \hbar} \mathcal{Q}_{\eta}-\frac{e}{c} \vec{A}^{2}(x)\right) \times \vec{p}\right) \cdot \vec{\Theta}=(\vec{\nabla} \mathcal{V}(x) \times \vec{p}) \cdot \vec{\Theta},
$$

the noncommutative Pauli equation in the presence of a uniform magnetic field simply reads

$$
\left\{\frac{1}{2 m}\left(-\hbar^{2} \vec{\nabla}^{2}+\frac{i e \hbar}{c}(\vec{\nabla} \cdot \vec{A}+\vec{A} \cdot \vec{\nabla})+\frac{e^{2}}{c^{2}} \vec{A}^{2}\right)-\frac{\vec{L} \cdot \vec{\eta}}{2 m}-\frac{e \mathcal{Q}_{\eta}}{2 m c \hbar}+\mu_{B} \vec{\sigma} \cdot \vec{B}+\frac{e \mathcal{Q}_{\Theta}}{4 m c \hbar}\right\} \psi=i \hbar \frac{\partial}{\partial t} \psi
$$

Knowing that $\vec{\sigma}, \vec{L}$ are Hermitian and the magnetic field is real, and $\mathcal{Q}_{\Theta}^{*}$ is the adjoint of $\mathcal{Q}_{\Theta}$, the adjoint equation of equation (26) reads

$$
\begin{aligned}
\frac{1}{2 m}\left\{-\hbar^{2} \vec{\nabla}^{2} \psi^{\dagger}-\frac{i e \hbar}{c}(\vec{\nabla} \cdot \vec{A}+\vec{A} \cdot \vec{\nabla}) \psi^{\dagger}+\frac{e^{2}}{c^{2}} \vec{A}^{2} \psi^{\dagger}\right\}-\frac{\vec{L} \cdot \vec{\eta}}{2 m} \psi^{\dagger}-\frac{e \mathcal{Q}_{\eta}}{2 m c \hbar} \psi^{\dagger}+\mu_{B} \vec{\sigma} \cdot \vec{B} \psi^{\dagger}+\frac{e}{4 m c \hbar} \psi^{\dagger} \mathcal{Q}_{\Theta}^{*}= \\
=-i \hbar \frac{\partial \psi^{\dagger}}{\partial t} . \quad(27)
\end{aligned}
$$

Here $*, \dagger$ stand for the complex conjugation of the potentials, operators and for the wave-functions, respectively.

To find the continuity equation, we multiply equation 26 from left by $\psi^{\dagger}$ and equation 27 from the right by $\psi$, making the subtraction of these equations yields

$$
\frac{-\hbar^{2}}{2 m}\left\{\psi^{\dagger} \vec{\nabla}^{2} \psi-\left(\vec{\nabla}^{2} \psi^{\dagger}\right) \psi\right\}+\frac{i e \hbar}{2 m c}\left\{\psi^{\dagger}(\vec{\nabla} \cdot \vec{A}+\vec{A} \cdot \vec{\nabla}) \psi+\left[(\vec{\nabla} \cdot \vec{A}+\vec{A} \cdot \vec{\nabla}) \psi^{\dagger}\right] \psi\right\}
$$

after some minor simplefications, we have

$$
\frac{-\hbar}{2 m} \operatorname{div}\left\{\psi^{\dagger} \vec{\nabla} \psi-\psi \vec{\nabla} \psi^{\dagger}\right\}+\frac{i e}{m c} \operatorname{div}\left\{\vec{A} \psi^{\dagger} \psi\right\}+\frac{e}{4 m c \hbar^{2}}\left(\psi^{\dagger} \mathcal{Q}_{\Theta} \psi-\psi^{\dagger} \mathcal{Q}_{\Theta}^{*} \psi\right)=i \frac{\partial}{\partial t} \psi^{\dagger} \psi
$$

This will be recognized as the deformed continuity equation. The obtained equation (29) contains a new quantity, which is the deformation due to the effect of the phase-space noncommutativity on the Pauli equation.

The third term on the left-hand side, which is the deformation quantity, can be simplified as follows

$$
\frac{i e}{4 m c \hbar^{2}}\left(\psi^{\dagger} \mathcal{Q}_{\Theta} \psi-\psi^{\dagger} \mathcal{Q}_{\Theta}^{*} \psi\right)=\frac{i e}{4 m c \hbar^{2}}\left(\psi^{\dagger}(\mathcal{V}(x) \star \psi)-\left(\psi^{\dagger} \star \mathcal{V}(x)\right) \psi\right),
$$

using the propriety $(\vec{a} \times \vec{b}) \cdot \vec{c}=\vec{a} \cdot(\vec{b} \times \vec{c})=\vec{b} \cdot(\vec{c} \times \vec{a})$, we must also pay attention to the order, $\psi^{\dagger}$ is the first and $\psi$ the second factor, we have

$$
\frac{i e}{4 m c \hbar^{2}}\left(\psi^{\dagger} \mathcal{Q}_{\Theta} \psi-\psi^{\dagger} \mathcal{Q}_{\Theta}^{*} \psi\right)=\frac{e}{8 m c \hbar^{2}} \operatorname{div} \mathcal{V}(x)\left(\vec{\Theta} \times \vec{\nabla}\left(\psi^{\dagger} \psi\right)\right)=\operatorname{div} \vec{\xi}^{n c}
$$

Using the following identity also gives the same equation as above [6]

$$
v^{\dagger}(\vec{\pi} \tau)-(\vec{\pi} v)^{\dagger} \tau=-i \hbar \vec{\nabla}\left(v^{\dagger} \tau\right)
$$

where $v, \tau$ are arbitrary two-component spinor. Noting that $\vec{A}$ does not appear on the right-hand side of the identity; and that this identity is related to the fact that $\vec{\pi}$ is Hermitian.

It is evident that the noncommutativity affects the current density, and the deformation quantity may apear as a correction to it. The deformed current density satisfies the current conservation, which means that we have a conservation of the continuity equation in the noncommutative phase-space. Equation (29) may be contracted as

$$
\frac{\partial \rho}{\partial t}+\vec{\nabla} \cdot \vec{j}^{n c}=0
$$

where

$$
\rho=\psi^{\dagger} \psi=|\psi|^{2}
$$


is the probability density and

$$
\vec{j}^{n c}=\vec{j}+\vec{\xi}^{n c}=\frac{-i \hbar}{2 m}\left\{\psi^{\dagger} \vec{\nabla} \psi-\psi \vec{\nabla} \psi^{\dagger}\right\}-\frac{e}{m c}\left\{\vec{A} \psi^{\dagger} \psi\right\}+\vec{\xi}^{n c},
$$

is the deformed current density of the electrons. The deformation quantity is

$$
\vec{\xi}^{n c}=\frac{e}{8 m c \hbar^{2}} \mathcal{V}(x)\left(\vec{\Theta} \times \vec{\nabla}\left(\psi^{\dagger} \psi\right)\right)=\frac{e}{8 m c \hbar^{2}}\left(2 \vec{p} \cdot \vec{A}-\frac{1}{2 \hbar} \mathcal{Q}_{\eta}-\frac{e}{c} \vec{A}^{2}\right)\left(\vec{\Theta} \times \vec{\nabla}\left(\psi^{\dagger} \psi\right)\right) .
$$

Furthermore, the deformed continuity equation for all orders of $\Theta$ is proportional to the magnetic field $\vec{B}$. In fact, one can explicitly calculate the conserved current for all orders of $\Theta$ in the case of a non-constant magnetic field, thus using equation (24), we have

$$
\frac{\partial \rho}{\partial t}+\vec{\nabla} \cdot \vec{j}+\frac{i e}{4 m c \hbar^{2}}\left\{\psi^{\dagger}(\mathcal{V}(x) \star \psi)-\left(\psi^{\dagger} \star \mathcal{V}(x)\right) \psi\right\}=0,
$$

we calculate the $n^{\text {th }}$ order term in the general deformed continuity equation (37) as follows

$$
\begin{gathered}
\psi^{\dagger}(\mathcal{V}(x) \star \psi)-\left.\left(\psi^{\dagger} \star \mathcal{V}(x)\right) \psi\right|_{n^{t h}}=\frac{1}{n !}\left(\frac{i}{2}\right)^{n} \Theta^{a_{1} b_{1}} \ldots \Theta^{a_{n} b_{n}} \\
\times\left(\psi^{\dagger} \partial_{a_{1}} \ldots \partial_{a_{k}} \mathcal{V}(x) \partial_{b_{1}} \ldots \partial_{b_{k}} \psi-\partial_{a_{1}} \ldots \partial_{a_{k}} \psi^{\dagger}\left(\partial_{b_{1}} \ldots \partial_{b_{k}} \mathcal{V}(x)\right) \psi+(-1)^{n} c c .\right) .
\end{gathered}
$$

We note the absence of the magnetization current term in equation (35), as in commutative case when this was asserted by authors [1, 4, 8, 13, 16, 27, where at first, they attempted to cover this deficiency by explaining how to derive this additional term from the non-relativistic limit of the relativistic Dirac probability current density. Then, Nowakowski and others [6] provided a superb explanation of how to extract this term through the non-relativistic Pauli equation itself.

Knowing that, in a commutative background, the magnetization current $\vec{j}_{M}$ from the probability current of the Pauli equation is proportional to $\vec{\nabla} \times\left(\psi^{\dagger} \vec{\sigma} \psi\right)$. However, the existence of such an additional term is important and it should be discussed when talking about the probability current of spin- $1 / 2$ particles. In following, we try to derive the current magnetization in a noncommutative background without changing the continuity equation, and seek if such an additional term is affected by the noncommutativity or not.

\subsection{DeRivation of THE MAGNetization CURRENT}

At first, it must be clarified that the authors Nowakowski and others (2011) in [4, 6 derived the non-relativistic current density for a spin- $1 / 2$ particle using minimally coupled Pauli equation. In contrast, Wilkes, J. M (2020) in [39] derived the non-relativistic current density for a free spin-1/2 particle using directly free Pauli equation. However, we show here that the current density can be derived from the minimally coupled Pauli equation in a noncommutative phase-space.

Starting with the noncommutative minimally coupled Pauli equation written in the form

$$
\mathcal{H}_{\text {Pauli }}^{n c} \psi=\frac{1}{2 m}\left(\vec{\sigma} \cdot \hat{\vec{\pi}}^{n c}\right)^{2} \psi=i \hbar \frac{\partial}{\partial t} \psi,
$$

we multiply the above equation from left by $\psi^{\dagger}$ and the adjoint equation of equation 39 from the right by $\psi$, the subtraction of these equations yields the following continuity equation

$$
2 m\left\{\left(\left(\vec{\sigma} \cdot \hat{\vec{\pi}}^{n c}\right)^{2} \psi\right)^{\dagger} \psi-\psi^{\dagger}\left(\vec{\sigma}^{\cdot \hat{\vec{\pi}}^{n c}}\right)^{2} \psi\right\}=i \hbar\left(\psi^{\dagger} \frac{\partial \psi}{\partial t}+\psi \frac{\partial \psi^{\dagger}}{\partial t}\right)
$$

noting that the noncommutativity of $\pi^{n c}$ has led us to express the two terms as follows

$$
\frac{i}{2 m \hbar} \sum_{i, j}\left\{\left(\hat{\pi}_{i}^{n c} \hat{\pi}_{j}^{n c} \psi\right)^{\dagger} \sigma_{j} \sigma_{i} \psi-\psi^{\dagger} \sigma_{i} \sigma_{j}\left(\hat{\pi}_{i}{ }^{n c} \hat{\pi}_{j}{ }^{n c} \psi\right)\right\}=\frac{\partial \rho}{\partial t} .
$$

While with only $p_{i}$, we would have no reason for preferring $p_{i} p_{j} \psi$ over $p_{j} p_{i} \psi$.

It is easy to verify that the identity (32) remains valid for $\vec{\pi}^{n c}$ because of the fact that $\vec{\pi}^{n c}$ is Hermitian. Therefore, through identity $(32)$, we have

$$
\frac{-1}{2 m} \sum_{i, j} \nabla_{i}\left\{\left(\hat{\pi}_{j}^{n c} \psi\right)^{\dagger} \sigma_{j} \sigma_{i} \psi+\psi^{\dagger} \sigma_{i} \sigma_{j}\left(\hat{\pi}_{j}{ }^{n c} \psi\right)\right\}+\frac{i}{2 m \hbar} \sum_{i, j}\left\{\left(\hat{\pi}_{j}{ }^{n c} \psi\right)^{\dagger} \sigma_{j} \sigma_{i}\left(\hat{\pi}_{i}{ }^{n c} \psi\right)-\left(\hat{\pi}_{i}{ }^{n c} \psi\right)^{\dagger} \sigma_{i} \sigma_{j}\left(\hat{\pi}_{j}{ }^{n c} \psi\right)\right\}=\frac{\partial \rho}{\partial t},
$$


then

$$
\frac{-1}{2 m} \sum_{i, j} \nabla_{i}\left\{\left(\hat{\pi}_{j}^{n c} \psi\right)^{\dagger} \sigma_{j} \sigma_{i} \psi+\psi^{\dagger} \sigma_{i} \sigma_{j}\left(\hat{\pi}_{j}^{n c} \psi\right)\right\}=\frac{\partial \rho}{\partial t} .
$$

Knowing that the $2^{\text {nd }}$ sum in equation 42 gives zero by swapping $i$ and $j$ for one of the sums, then the probability current vector from the above continuity equation is

$$
j_{i}=\frac{1}{2 m} \sum_{j}\left\{\left(\hat{\pi}_{j}^{n c} \psi\right)^{\dagger} \sigma_{j} \sigma_{i} \psi+\psi^{\dagger} \sigma_{i} \sigma_{j}\left(\hat{\pi}_{j}^{n c} \psi\right)\right\}
$$

Using the property (15), equation 44) becomes

$$
j_{i}=\frac{1}{2 m} \sum_{j}\left\{\left(\hat{\pi}_{j}^{n c} \psi\right)^{\dagger} \psi+\psi^{\dagger}\left(\hat{\pi}_{j}^{n c} \psi\right)+i \sum_{k}\left[\epsilon_{j i k}\left(\hat{\pi}_{j}^{n c} \psi\right)^{\dagger} \sigma_{k} \psi+\epsilon_{i j k} \psi^{\dagger} \sigma_{k}\left(\hat{\pi}_{j}^{n c} \psi\right)\right]\right\},
$$

with $\epsilon_{j i k}=-\epsilon_{i j k}$, and using one more time identity [32, we find (this is similar to investigation by [6] in the case of commutative phase-space)

$$
j_{i}=\frac{1}{2 m}\left[\left(\hat{p}_{j}^{n c} \psi\right)^{\dagger} \psi-\frac{e}{c}\left(A_{j}^{n c} \psi\right)^{\dagger} \psi+\psi^{\dagger} \hat{p}_{j}{ }^{n c} \psi-\frac{e}{c} \psi^{\dagger} A_{j}^{n c} \psi\right]+\frac{\hbar}{2 m} \sum_{j, k} \epsilon_{i j k} \nabla_{j}\left(\psi^{\dagger} \sigma_{k} \psi\right) .
$$

In the right-hand side of the above equation, the first term will be interpreted as the noncommutative current vector $\vec{j}^{n c}$ given by equation $[36$, and the second term is the requested additional term, namely current magnetization $\vec{j}_{M}$, where

$$
\underset{M_{i}}{j}=\frac{\hbar}{2 m}\left(\vec{\nabla} \times\left(\psi^{\dagger} \vec{\sigma} \psi\right)\right)_{i}
$$

Furthermore, $\vec{j}_{M}$ can also be shown to be a part of the conserved Noether current [40], resulting from the invariance of the Pauli Lagrangian under the global phase transformation $\mathrm{U}(1)$.

What can be concluded here is that the magnetization current is not affected by the noncommutativity, perhaps because the spin operator could not be affected by the noncommutativity. This is in contrast to what was previously found around the current density, which showed a great influence of the noncommutativity.

\section{Noncommutative Semi-classical Partition Function}

In this part of our work, we investigate the magnetization and the magnetic susceptibility quantities of our Pauli system using the partition function in a noncommutative phase-space. We concentrate, at first, on the calculation of the semi-classical partition function. Our studied system is semi-classical, so our system is not completely classical but contains a quantum interaction concerning the spin, therefore, the noncommutative partition function is separable into two independent parts as follows

$$
\mathcal{Z}^{n c}=Z_{\text {clas }}^{n c} Z_{n c l}
$$

where $Z_{n c l}$ is the non-classical part of the partition function. To study our noncommutative classical partition function, we assume that the passage between noncommutative classical mechanics and noncommutative quantum mechanics can be realized through the following generalized Dirac quantization condition [41-43]

$$
\{f, g\}=\frac{1}{i \hbar}[F, G]
$$

where $F, G$ stand for the operators associated with classical observables $f, g$ and $\{$,$\} stands for Poisson bracket.$ Using the condition above, we obtain from Eq. (5)

$$
\left\{x_{j}^{n c}, x_{k}^{n c}\right\}=\frac{1}{2} \epsilon_{j k l} \Theta_{l}, \quad\left\{p_{j}^{n c}, p_{k}^{n c}\right\}=\frac{1}{2} \epsilon_{j k l} \eta_{l}, \quad\left\{x_{j}^{n c}, p_{k}^{n c}\right\}=\delta_{j k}+\frac{1}{4 \hbar^{2}} \Theta_{j l} \eta_{k l}=\delta_{j k} \quad,(j, k, l=1,2,3) .
$$

It is worth mentioning that in terms of the classical limit, $\frac{\Theta \eta}{4 \hbar^{2}} \ll 1$ (check ref. [42]), thus $\left\{x_{j}^{n c}, p_{k}^{n c}\right\}=\delta_{j k}$. Now based on the proposal that noncommutative observables $F^{n c}$ correspond to the commutative one $F(x, p)$ can be defined by [44, 45]

$$
F^{n c}=F\left(x^{n c}, p^{n c}\right),
$$

and for non-interacting particles, the classical partition function in the canonical ensemble in a noncommutative phase-space is given by the following formula [41, 42]

$$
Z_{\text {clas }}^{n c}=\frac{1}{N !(2 \pi \tilde{\hbar})^{3 N}} \int e^{-\beta \mathcal{H}_{\text {clas }}^{n c}(x, p)} d^{3 N} x^{n c} d^{3 N} p^{n c},
$$


which is written for an $N$ particles. $\frac{1}{N !}$ is the Gibbs correction factor, considered due to accounting for indistinguishability, which means that there are $N$ ! ways of arranging $N$ particles at $N$ sites. $\tilde{\hbar} \sim \triangle x^{n c} \triangle p^{n c}$, with $\frac{1}{\hbar^{3}}$ is a factor that makes the volume of the noncommutative phase-space dimensionless.

$\beta$ defined as $\frac{1}{K_{B} T}, K_{B}$ is the Boltzmann constant, where $K_{B}=1.38 \times 10^{-23} \mathrm{JK}^{-1}$. The Helmholtz free energy is

$$
F=-\frac{1}{\beta} \ln \mathcal{Z}
$$

we may derive the magnetization as follows

$$
\langle M\rangle=-\frac{\partial F}{\partial B} .
$$

For a single particle, the noncommutative classical partition function is then

$$
Z_{\text {clas }, 1}^{n c}=\frac{1}{\tilde{h}^{3}} \int e^{-\beta \mathcal{H}_{\text {Clas }}^{n c}(x, p)} d^{3} x^{n c} d^{3} p^{n c},
$$

where $d^{3}$ is a shorthand notation serving as a reminder that the $x$ and $p$ are vectors in a three-dimensional phase-space. The relation between equation 52$)$ and $(55)$ is given by the following formula

$$
Z_{\text {clas }}^{n c}=\frac{\left(Z_{\text {clas }, 1}^{n c}\right)^{N}}{N !}
$$

Knowing that, using equation (6), we have

$$
d^{3} x^{n c} d^{3} p^{n c}=\left(1-\frac{\Theta \eta}{8 \hbar^{2}}\right) d^{3} x d^{3} p
$$

furthermore, using uncertainty principle and according to the third equation of equation (4), we deduce

$$
\tilde{h}^{3}=h^{3}\left(1+\frac{3 \Theta \eta}{4 \hbar^{2}}\right)+\mathcal{O}\left(\Theta^{2} \eta^{2}\right)
$$

Unlike other works such as [41], where the researchers used a different formula for Planck's constant $\tilde{h}_{11}=$ $\tilde{h}_{22} \neq \tilde{h}_{33}$, which led to a different formula of $\tilde{h}^{3}$.

For an electron with a spin in interaction with an electromagnetic potential, once the magnetic field $\vec{B}$ be in the z-direction, and by equation $\left[19\right.$, bear in mind that $\left[\vec{p}^{n c}, \vec{A}^{n c}\right]=0$, then for the sake of simplicity, the noncommutative Pauli Hamiltonian from equation 233 takes the form

$$
\mathcal{H}_{\text {Pauli }}\left(x^{n c}, p^{n c}\right)=\frac{1}{2 m}\left\{\left(\vec{p}^{n c}\right)^{2}-2 \frac{e}{c} \vec{p}^{n c} \cdot \vec{A}^{n c}+\left(\frac{e}{c}\right)^{2}\left(\vec{A}^{n c}\right)^{2}\right\}+\mu_{B} \hat{\sigma}_{z} B
$$

We split the noncommutative Pauli Hamiltonian as $\mathcal{H}_{\text {Pauli }}^{n c}=\mathcal{H}_{c l a}^{n c}+\mathcal{H}_{n c l, \sigma}$, with $\mathcal{H}_{n c l, \sigma}=\mu_{B} \hat{\sigma}_{z} B$. It is easy to verify that

$$
\begin{gathered}
\left(\vec{p}^{n c}\right)^{2}=\left(p_{x}^{n c}\right)^{2}+\left(p_{y}^{n c}\right)^{2}+\left(p_{z}^{n c}\right)^{2}=p_{x}^{2}+p_{y}^{2}+p_{z}^{2}-\frac{\eta}{2 \hbar} L_{z}+\frac{\eta^{2}}{16 \hbar^{2}}\left(x^{2}+y^{2}\right) \\
\vec{p}^{n c} \cdot \vec{A}=p_{x}^{n c} A_{x}^{n c}+p_{y}^{n c} A_{y}^{n c}=\frac{B}{2}\left\{-\frac{\Theta}{4 \hbar}\left(p_{x}^{2}+p_{y}^{2}\right)-\frac{\eta}{4 \hbar}\left(y^{2}+x^{2}\right)+\left(1+\frac{\Theta \eta}{16 \hbar^{2}}\right) L_{z}\right\}, \\
\left(\vec{A}^{n c}\right)^{2}=\left(A_{x}^{n c}\right)^{2}+\left(A_{y}^{n c}\right)^{2}=\frac{B^{2}}{4}\left\{x^{2}+y^{2}-\frac{\Theta}{2 \hbar} L_{z}+\frac{\Theta^{2}}{16 \hbar^{2}}\left(p_{x}^{2}+p_{y}^{2}\right)\right\}
\end{gathered}
$$

Using the three equations above, our noncommutative classical Hamiltonian becomes

$$
\mathcal{H}_{c l a}^{n c}=\frac{1}{2 \tilde{m}}\left(p_{x}^{2}+p_{y}^{2}\right)+\frac{1}{2 m} p_{z}^{2}-\tilde{\omega} L_{z}+\frac{1}{2} \tilde{m} \tilde{\omega}^{2}\left(x^{2}+y^{2}\right),
$$

where $L_{z}=p_{y} x-p_{x} y=\left(x_{i} \times p_{i}\right)_{z}$, and

$$
\tilde{m}=\frac{m}{\left(1+\frac{e B \Theta}{8 c \hbar}\right)^{2}}, \tilde{\omega}=\frac{c \eta+2 e \hbar B}{4 c \hbar \tilde{m}\left(1+\frac{e B \Theta}{c 8 \hbar}\right)} \text { and } \frac{1}{2} \tilde{m} \tilde{\omega}^{2}=\frac{1}{2 m}\left(\frac{\eta e B}{4 c \hbar}+\frac{\eta^{2}}{16 \hbar^{2}}+\frac{e^{2} B^{2}}{c^{2} 4}\right) .
$$


Now, following the definition given in equation (55), we express the single particle noncommutative classical partition function as

$$
Z_{\text {clas }, 1}^{n c}=\frac{1}{\tilde{h}^{3}} \int e^{-\beta\left[\frac{1}{2 \tilde{m}}\left(p_{x}^{2}+p_{y}^{2}\right)+\frac{1}{2 m} p_{z}^{2}-\tilde{\omega} L_{z}+\frac{1}{2} \tilde{m} \tilde{\omega}^{2}\left(x^{2}+y^{2}\right)\right]} d^{3} x^{n c} d^{3} p^{n c} .
$$

It should be noted that we want to factorize our Hamiltonian into momentum and position terms. This is not always possible when there are matrices (or operators) in the exponent. However, within the classical limit, it is possible. Otherwise, to separate the operators in the exponent, we use the Baker-Campbell-Hausdorff (BCH) formula given by (first few terms)

$$
e^{[\hat{A}+\hat{B}]}=e^{[\hat{A}]} e^{[\hat{B}]} e^{\left[-\frac{1}{2}[\hat{A}, \hat{B}]\right]} e^{\frac{1}{6}(2[\hat{A},[\hat{A}, \hat{B}]]+[\hat{B},[\hat{A}, \hat{B}]])} \ldots
$$

We can now start to replace some of the operators in the exponent

$$
Z_{\text {clas }, 1}^{n c}=\frac{1}{\tilde{h}^{3}} \int e^{-\beta\left[\frac{1}{2 \tilde{m}}\left(p_{x}^{2}+p_{y}^{2}\right)+\frac{1}{2 m} p_{z}^{2}\right]} e^{-\beta\left[\frac{1}{2} \tilde{m} \tilde{\omega}^{2}\left(x^{2}+y^{2}\right)\right]} e^{\beta \tilde{\omega} L_{z}} d^{3} p^{n c} d^{3} x^{n c} .
$$
obtain

We should expand exponentials containing $\tilde{\omega}$, and by considering terms up to the second-order of $\tilde{\omega}$, we

$$
Z_{\text {clas }, 1}^{n c}=\frac{1}{\tilde{h}^{3}} \int e^{-\frac{\beta}{2}\left[\frac{p_{x}^{2}+p_{y}^{2}}{\tilde{m}}+\frac{p_{z}^{2}}{m}\right]}\left(1+\beta \tilde{\omega} L_{z}+\frac{1}{2} \beta^{2} \tilde{\omega}^{2} L_{z}^{2}\right)\left(1-\beta \tilde{\omega}^{2} \frac{\tilde{m}}{2}\left(x^{2}+y^{2}\right)\right) d^{3} p^{n c} d^{3} x^{n c},
$$

therefore, we have the appropriate expression for $Z_{c l a s, 1}^{n c}$

$$
\begin{gathered}
Z_{c l a s, 1}^{n c}=\frac{1-\frac{7 \Theta \eta}{8 \hbar^{2}}}{h^{3}} \int e^{-\frac{\beta}{2}\left[\frac{p_{x}^{2}+p_{y}^{2}}{\tilde{m}}+\frac{p_{z}^{2}}{m}\right]} d^{3} p d^{3} x+\frac{\left(1-\frac{7 \Theta \eta}{8 \hbar^{2}}\right) \beta \tilde{\omega}}{h^{3}} \int e^{-\frac{\beta}{2}\left[\frac{p_{x}^{2}+p_{y}^{2}}{\tilde{m}}+\frac{p_{z}^{2}}{m}\right]} L_{z} d^{3} p d^{3} x \\
+\frac{\left(1-\frac{7 \Theta \eta}{8 \hbar^{2}}\right) \beta^{2} \tilde{\omega}^{2}}{2 h^{3}} \int e^{-\frac{\beta}{2}\left[\frac{p_{x}^{2}+p_{y}^{2}}{\tilde{m}}+\frac{p_{z}^{2}}{m}\right]} L_{z}^{2} d^{3} p d^{3} x-\frac{\left(1-\frac{7 \Theta \eta}{8 \hbar^{2}}\right) \beta \tilde{\omega}^{2}}{2 h^{3}} \int e^{-\frac{\beta}{2}\left[\frac{p_{x}^{2}+p_{y}^{2}}{\tilde{m}}+\frac{p_{z}^{2}}{m}\right]}\left(x^{2}+y^{2}\right) d^{3} p d^{3} x .
\end{gathered}
$$

In the right-hand side of the above equation, it is easy to check that the second integral goes to zero and the third and last integrals cancel each other, and thus we obtain

$$
Z_{\text {clas }, 1}^{n c}=\frac{1-\frac{7 \Theta \eta}{8 \hbar^{2}}}{h^{3}} \int e^{-\frac{\beta}{2}\left[\frac{p_{x}^{2}+p_{y}^{2}}{\tilde{m}}+\frac{p_{z}^{2}}{m}\right]} d^{3} p d^{3} x
$$

Using the integral of Gaussian function $\int e^{-a x^{2}} d x=\sqrt{\frac{\pi}{a}}$, we have

$$
Z_{\text {clas }, 1}^{n c}=\frac{1-\frac{7 \Theta \eta}{8 \hbar^{2}}}{h^{3}} \int d^{3} x \int e^{-\frac{\beta}{2}\left[\frac{p_{x}^{2}+p_{y}^{2}}{\tilde{m}}+\frac{p_{z}^{2}}{m}\right]} d^{3} p=\frac{V}{\Lambda^{3}} \frac{1-\frac{7 \Theta \eta}{8 \hbar^{2}}}{\left(1+\frac{e B \Theta}{8 c \hbar}\right)^{2}}
$$

where $V, \Lambda=h\left(2 m \pi K_{B} T\right)^{-\frac{1}{2}}$ are the volume and the thermal de Broglie wavelength, respectively. The non-classical partition function using $\mathcal{H}_{n c l, \sigma}$ is

$$
Z_{n c l}=Z_{n c l, 1}^{N}=\left(\sum_{\sigma_{z}= \pm 1} e^{\beta \mu_{B} \hat{\sigma}_{z} B}\right)^{N}=2^{N} \cosh ^{N}\left(\beta \mu_{B} B\right) .
$$

Finally, the Pauli partition function for a system of $N$ particles in a three-dimensional noncommutative phase-spaces is

$$
\mathcal{Z}^{n c}=\frac{(2 V)^{N}}{\Lambda^{3 N} N !} \frac{\left(1-\frac{7 \Theta \eta}{8 \hbar^{2}}\right)^{N} \cosh ^{N}\left(\beta \mu_{B} B\right)}{\left(1+\frac{e B \Theta}{8 c \hbar}\right)^{2 N}}
$$


In the limit of the noncommutativity, i.e. $\Theta \rightarrow 0, \eta \rightarrow 0$, the above expression of $\mathcal{Z}^{n c}$ tends to the result of $\mathcal{Z}$ in the usual commutative phase-space, which is

$$
\mathcal{Z}=\frac{(2 V)^{N}}{\Lambda^{3 N} N !} \cosh ^{N}\left(\beta \mu_{B} B\right) .
$$
thus

Using formulae (53) and (54), we find the magnetization in noncommutative and commutative phase-space,

$$
F^{n c}=-\frac{N}{\beta} \ln \frac{(2 V)}{\Lambda^{3}} \frac{\left(1-\frac{7 \Theta \eta}{8 \hbar^{2}}\right) \cosh \left(\beta \mu_{B} B\right)}{\left(1+\frac{e B \Theta}{8 c \hbar}\right)^{2}}+\frac{1}{\beta} \ln N !,
$$

we can use $\ln N !=N \ln N-N$ (Stirling's formula) to simplify further. The noncommutative magnetization is

$$
\left\langle M^{n c}\right\rangle=-\frac{\partial F^{n c}}{\partial B}=2 \frac{N}{\beta} \frac{e \Theta}{(8 c \hbar+e B \Theta)}+N \mu_{B} \tanh \left(\beta \mu_{B} B\right) .
$$

The commutative magnetization is

$$
\langle M\rangle=-\frac{\partial F}{\partial B}=N \mu_{B} \tanh \left(\beta \mu_{B} B\right),
$$

it is obvious that $\left.\left\langle M^{n c}\right\rangle\right|_{\Theta=0}=\langle M\rangle$. We may derive the magnetic susceptibility of electrons $\chi=\frac{1}{V} \frac{\partial\langle M\rangle}{\partial B}$ in a noncommutative phase-space using the magnetization $(76)$ by

$$
\chi^{n c}=-2 \frac{N}{V \beta} \frac{(e \Theta)^{2}}{(8 c \hbar+e B \Theta)^{2}}+\frac{N}{V} \beta \mu_{B}^{2}\left(1-\tanh ^{2}\left(\beta \mu_{B} B\right)\right),
$$

where the commutative magnetic susceptibility $\chi=\chi^{n c}(\Theta=0)$ is

$$
\chi=\frac{N}{V} \beta \mu_{B}^{2}\left(1-\tanh ^{2}\left(\beta \mu_{B} B\right)\right) .
$$

Finally, we conclude with the following special cases. Let us first consider $B=0$, then we have

$$
\langle M\rangle=0 ;\left\langle M^{n c}\right\rangle=2 \frac{N}{\beta} \frac{e \Theta}{8 c \hbar} ; \text { and } \chi^{n c}=-2 \frac{N}{V \beta} \frac{(e \Theta)^{2}}{(8 c \hbar)^{2}}+\frac{N}{V} \beta \mu_{B}^{2} .
$$

For $B \rightarrow \infty$ and $T=C^{s t}, \tanh \left(\beta \mu_{B} B\right)=1$, we obtain

$$
\left\langle M^{n c}\right\rangle=\langle M\rangle=N \mu_{B} ; \text { and } \chi^{n c}=\chi \sim 0 .
$$

As well when $T \rightarrow \infty, \beta \rightarrow 0$ (with $B$ be constant), $\tanh \left(\beta \mu_{B} B\right)=0$, we obtain

$$
\left\langle M^{n c}\right\rangle \rightarrow \infty,\langle M\rangle=0 \text {; and } \chi^{n c} \rightarrow \infty, \chi=0 .
$$

Armed with the partition function $\mathcal{Z}$, we can compute other important thermal quantities, such as the average energy $U=-\frac{\partial}{\partial \beta} \ln \mathcal{Z}$, the entropy $S=\ln \mathcal{Z}-\beta \frac{\partial}{\partial \beta} \ln \mathcal{Z}$ and the specific heat $C=\beta^{2} \frac{\partial^{2}}{\partial^{2} \beta} \ln \mathcal{Z}$. This is of course in both case of noncommutative and commutative phase-spaces.

\section{Conclusion}

In this work, we have studied the three-dimensional Pauli equation and the corresponding continuity equation for a spin-1/2 particle in the presence of an electromagnetic field in a noncommutative phase-space, considering constant and non-constant magnetic fields. It is shown that the non-constant magnetic field lifts the order of the noncommutativity parameter in both the Pauli equation and the corresponding continuity equation. Given the known absence of the magnetization current term in the continuity equation, even in the noncommutative phase-space as confirmed by our calculations, we extracted the magnetization current term from the Pauli equation itself without modifying the continuity equation. Furthermore, we found that the density current is conserved, which means that we have a conservation of the deformed continuity equation.

By using the classical treatment (within the classical limit), the magnetization and the magnetic susceptibility quantities are explicitly determined in both commutative and noncommutative phase-spaces through a semiclassical partition function of the Pauli system of the one-particle and N-particle systems in three dimensions. Furthermore, to see the behaviour of these deformed quantities, we carried out some special cases in commutative and noncommutative phase-spaces.

Finally, we can say that we successfully examined the influence of the noncommutativity on the problems in question, where the noncommutativity was introduced using both the three-dimensional Bopp-Shift transformation and the Moyal-Weyl product. Furthermore, the noncommutative corrections to the nonrelativistic Pauli equation and the continuity equation are also valid up to all orders in the noncommutative parameter. Our results' limits are in good agreement with those obtained by other authors as discussed and in the literature. 


\section{ACKNOWLEDGEMENTS}

The author would like to thank Dr. Mojtaba Najafizadeh for his valuable discussion on the classical partition function.

\section{REFERENCES}

[1] W. Greiner. Quantum Mechanics: An Introduction. Springer, Berlin, 4th edn., 2001.

[2] E. Ikenberry. Quantum Mechanics for Mathematicians and Physicists. Oxford, New York, 1st edn., 1962.

[3] A. Galindo, C. Sanchez del Rio. Intrinsic magnetic moment as a nonrelativistic phenomenon. American Journal of Physics 29(9):582 - 584, 1961. DOI:10.1119/1.1937856

[4] M. Nowakowski. The quantum mechanical current of the Pauli equation. American Journal of Physics 67(10):916 919, 1999. DOI:10.1119/1.19149.

[5] G. W. Parker. Spin current density and the hyperfine interaction in hydrogen. American Journal of Physics 52(1):36 - 39, 1984. DOI:10.1119/1.13846

[6] M. S. Shikakhwa, S. Turgut, N. K. Pak. Derivation of the magnetization current from the non-relativistic Pauli equation: A comment on "The quantum mechanical current of the Pauli equation" by Marek Nowakowski. American Journal of Physics 79(11):1177 - 1179, 2011. DOI:10.1119/1.3630931.

[7] W. B. Hodge, S. V. Migirditch, W. C. Kerr. Electron spin and probability current density in quantum mechanics. American Journal of Physics 82(7):681 - 690, 2014. DOI:10.1119/1.4868094.

[8] J. J. Sakurai. Advanced Quantum Mechanics. Reading, Mass.: Addison-Wesley Pub. Co., 1967.

[9] I. Haouam, L. Chetouani. The Foldy-Wouthuysen transformation of the Dirac equation in noncommutative Phase-Space. Journal of Modern Physics 9(11):2021 - 2034, 2018. DOI:10.4236/jmp.2018.911127.

[10] I. Haouam. The phase-space noncommutativity effect on the large and small wavefunction components approach at Dirac equation. Open Access Library Journal 5:e4108, 2018. DOI:10.4236/oalib.1104108.

[11] J. D. Bjorken, S. Drell. Relativistic Quantum Mechanics. McGraw-Hill, New York, 1964.

[12] L. L. Foldy, S. A. Wouthuysen. On the Dirac theory of spin 1/2 particles and its non-relativistic limit. Physical Review 78(1):29 - 36, 1950. DOI:10.1103/PhysRev.78.29.

[13] W. Pauli. Zur quantenmechanik des magnetischen elektrons. Zeitschrift für Physik 43:601 - 623, 1927. DOI:10.1103/PhysRev.78.29

[14] G. S, G. E. Uhlenbeck. Opmerking over de Spectra van Waterstof en Helium. Physica 5:266 - 270, 1925.

[15] G. E. Uhlenbeck, S. Goudsmit. Ersetzung der Hypothese vom unmechanischen Zwang durch eine Forderung bezüglich des inneren Verhaltens jedes einzelnen Elektrons. Die Naturwissenschaften 13(47).

[16] P. A. M. Dirac, R. H. Fowler. The quantum theory of the electron. Proceedings of the Royal Society of London Series A, Containing Papers of a Mathematical and Physical Character 117(778):610 - 624, 1928. DOI:10.1098/rspa.1928.0023

[17] A. Connes. Non-commutative differential geometry. Publications Mathématiques de l'Institut des Hautes Études Scientifiques 62(1):41 - 144, 1985. DOI:10.1007/BF02698807

[18] S. L. Woronowicz. Twisted SU (2) group. An example of a non-commutative differential calculus. Publications of the Research Institute for Mathematical Sciences 23(1):117 - 181, 1987. DOI:10.2977/prims/1195176848.

[19] A. Connes, M. R. Douglas, A. Schwarz. Noncommutative geometry and matrix theory. Journal of High Energy Physics 1998(JHEP02):003, 1998. DOI:10.1088/1126-6708/1998/02/003

[20] M. M. Sheikh-Jabbari. $C, P$, and $T$ invariance of noncommutative gauge theories. Physical Review Letters 84(23):5265 - 5268, 2000. DOI:10.1103/PhysRevLett.84.5265

[21] E. Di Grezia, G. Esposito, A. Funel, et al. Spacetime noncommutativity and antisymmetric tensor dynamics in the early Universe. Physical Review D 68(10):105012, 2003. DOI:10.1103/PhysRevD.68.105012.

[22] O. Bertolami, J. G. Rosa, C. M. L. de Aragão, et al. Noncommutative gravitational quantum well. Physical Review D 72(2):025010, 2005. DOI:10.1103/PhysRevD.72.025010.

[23] A. Das, H. Falomir, J. Gamboa, F. Méndez. Non-commutative supersymmetric quantum mechanics. Physics Letters $B$ 670(4-5):407 - 415, 2009. DOI:10.1016/j.physletb.2008.11.011.

[24] J. M. Gracia-Bondia. Notes on "quantum gravity" and noncommutative geometry. In New Paths Towards Quantum Gravity, pp. 3 - 58. Springer, 2010. DOI:10.1007/978-3-642-11897-5_1.

[25] P. Martinetti. Beyond the standard model with noncommutative geometry, strolling towards quantum gravity. vol. 634, p. 012001. IOP Publishing, 2015. DOI:10.1088/1742-6596/634/1/012001.

[26] N. Seiberg, E. Witten. String theory and noncommutative geometry. Journal of High Energy Physics 1999(JHEP09), 1999. DOI:10.1088/1126-6708/1999/09/032

[27] K. Christian. Quantum groups. Graduate texts in mathematics 155. Springer-Verlag, New York, 1995. 
[28] A. Connes, D. Kreimer. Renormalization in quantum field theory and the Riemann-Hilbert problem I: the Hopf algebra structure of graphs and the main theorem. Communications in Mathematical Physics 210(1):249 - 273, 2000. DOI:10.1007/s002200050779

[29] A. Connes, D. Kreimer. Renormalization in quantum field theory and the Riemann-Hilbert problem II: The $\beta$-function, diffeomorphisms and the renormalization group. Communications in Mathematical Physics 216(1):215 241, 2001. DOI:10.1007/PL00005547.

[30] A. Tanasa, F. Vignes-Tourneret. Hopf algebra of non-commutative field theory. Journal of Noncommutative Geometry 2(1), 2008.

[31] S. M. Carroll, J. A. Harvey, V. A. Kostelecký, et al. Noncommutative field theory and Lorentz violation. Physical Review Letters 87(14):141601, 2001. DOI:10.1103/PhysRevLett.87.141601

[32] R. J. Szabo. Quantum field theory on noncommutative spaces. Physics Reports 378(4):207 - 299, 2003. DOI:10.1016/S0370-1573(03)00059-0

[33] I. Haouam. On the Fisk-Tait equation for spin-3/2 fermions interacting with an external magnetic field in noncommutative space-time. Journal of Physical Studies 24(1):1801, 2020. DOI:10.30970/jps.24.1801.

[34] K. Li, J. Wang, C. Chen. Representation of noncommutative phase space. Modern Physics Letters A 20(28):2165 2174, 2005. DOI:10.1142/S0217732305017421.

[35] I. Haouam. Analytical solution of $(2+1)$ dimensional Dirac equation in time-dependent noncommutative phase-space. Acta polytechnica 60(2):111 - 121, 2020. DOI:10.14311/AP.2020.60.0111

[36] I. Haouam. On the noncommutative geometry in quantum mechanics. Journal of Physical Studies 24(2):2002, 2020. DOI:10.30970/jps.24.2002

[37] I. Haouam. The non-relativistic limit of the DKP equation in non-commutative phase-space. Symmetry 11(2):223, 2019. DOI:10.3390/sym11020223

[38] I. Haouam. Continuity equation in presence of a non-local potential in non-commutative phase-space. Open Journal of Microphysics 9(3):15 - 28, 2019. DOI:10.4236/ojm.2019.93003.

[39] J. M. Wilkes. The Pauli and Lévy-Leblond equations, and the spin current density. European Journal of Physics 41(3):035402, 2020. DOI:10.1088/1361-6404/ab7495.

[40] M. E. Peskin, D. V. Schroeder. An Introduction to Quantum Field Theory. Reading, Mass.: Addison-Wesley Pub. Co., New York, 1995.

[41] M. Najafizadeh, M. Saadat. Thermodynamics of classical systems on noncommutative phase space. Chinese Journal of Physics 51(1):94 - 102, 2013. DOI:10.6122/CJP.51.94

[42] W. Gao-Feng, L. Chao-Yun, L. Zheng-Wen, et al. Classical mechanics in non-commutative phase space. Chinese Physics C 32(5):338, 2008. DOI:10.1088/1674-1137/32/5/002.

[43] A. E. F. Djemai, H. Smail. On quantum mechanics on noncommutative quantum phase space. Communications in Theoretical Physics 41(6):837, 2004. DOI:10.1088/0253-6102/41/6/837.

[44] M. Chaichian, M. M. Sheikh-Jabbari, A. Tureanu. Hydrogen atom spectrum and the Lamb shift in noncommutative QED. Physical Review Letters 86(13):2716 - 2719, 2001.

[45] S. Biswas. Bohr-van Leeuwen theorem in non-commutative space. Physics Letters A 381(44):3723 - 3725, 2017. DOI:10.1016/j.physleta.2017.10.003 